\title{
Heart rate variability analysis using robust period detection
}

\author{
Jørgen H Skotte ${ }^{*}$ and Jesper Kristiansen
}

\author{
* Correspondence: jhs@nrcwe.dk \\ National Research Centre for the \\ Working Environment, Lersø \\ Parkallé 105, DK-2100 Copenhagen, \\ Denmark
}

\begin{abstract}
Objective: Heart rate variability (HRV) analysis, which is an important tool for activity assessment of the cardiac autonomic nervous system, very often includes the estimation of power spectra for series of interbeat intervals (IBI). Ectopic beats and artifacts have a destructive effect on the standard methods (Fourier transform, FFT) for frequency analysis. This study investigates an alternative method for calculation of the periodogram using a robust period detection (RPD).

Method: Error free IBI series of 5 minutes for 221 subjects during one day were artificially distorted by randomly changing $|\mathrm{B}|$ values by $\pm 15-40 \%$. The low to high frequency rate (LF/HF) were calculated from periodograms estimated by the FFT, RPD and Lomb (LSP) methods for both error free and distorted series and for series with removed beats. Log transformed LF/HF values for series with distorted/removed beats were compared to undistorted values by linear regression.

Results: For series with $10 \%$ of distorted $|\mathrm{B}|$ values the regression analysis between distorted and undistorted series showed a goodness of fit, coefficient and intercept of $0.98,0.94$ and 0.02 , respectively. In comparison, the values of these parameters were $(0.34,0.46,-1.61)$ and $(0.28,0.42,-1.32)$ for the FFT and LSP methods, respectively. Similarly, the comparison between series with removed and undistorted beats yielded goodness of fit, coefficient and intercept of $(0.98,0.96,-0.01),(0.93$, $0.78,-0.02)$ and $(0.98,0.95,0.19)$ for RPD, FFT and LSP, respectively.

Conclusion: The RPD method demonstrated superior performance compared to the FFT and LSP method by estimation of power spectral characteristics for HRV analysis.

Keywords: Heart rate variability, Robust periodogram, Power spectrum, HRV, FFT, RPD
\end{abstract}

\section{Background}

Heart rate variability (HRV) is a method that is increasingly used to assess autonomic cardiac regulation of the heart in subjects during free-living conditions. Briefly, the beat-to-beat variation in heart rate (HR) is a result of the opposing influences of the parasympathetic and sympathetic divisions of the autonomic nervous system (see, for example, $[1,2])$. Due to fact that it takes longer for cardiac pacemaker cells to respond to sympathetic neural signals compared to parasympathetic signals, the relative activity in these two divisions of the autonomic nervous system can be disentangled by analyzing the frequency content of the interbeat interval time series [3]. Thus, the high frequency $(\mathrm{HF}, 0.15-0.4 \mathrm{~Hz}$ ) power in the interbeat interval series reflects parasympathetic influence on cardiac regulation, while the low frequency power

(c) 2014 Skotte and Kristiansen; licensee BioMed Central Ltd. This is an Open Access article distributed under the terms of the Creative Commons Attribution License (http://creativecommons.org/licenses/by/4.0), which permits unrestricted use, distribution, and reproduction in any medium, provided the original work is properly credited. The Creative Commons Public Domain Dedication waiver (http://creativecommons.org/publicdomain/zero/1.0/) applies to the data made available in this article, unless otherwise stated. 
(LF, 0.04-0.15 Hz) predominantly reflects sympathetic modulation of the cardiac rhythm. The ratio between the low and high frequency power (LF/HF) is interpreted as the balance between the sympathetic and parasympathetic modulation of cardiac rhythm [4-6], and is widely used because it captures essential physiological information in a single parameter [7-9].

Metrics for assessing HRV are generally based on either time-domain (time series) or frequency-domain analysis. Several advanced filtering techniques (linear and nonlinear) have been described in the literature, but traditionally the fast Fourier transform algorithm (FFT) is a central part of the frequency-domain methods [10,11]. Raw heart rate data consist of series of interbeat values (tachogram, distances between peaks in the QRS complex, RR data), which often contains errors or irregularities caused by artefacts or ectopic beats. Heart rate (HR) data recorded during everyday life including work hours often contain considerable amount of erroneous detected beats, typically during periods with intense movement. It is well known that the FFT analysis is highly sensitive to artefacts and even a small rate of faulty beats e.g. 1- $2 \%$ will cause bias in the calculation of the power spectrum $[12,13]$, hence it is mandatory to detect and remove artefacts. After the error correction process, the RR data must be interpolated and resampled at a fixed rate (e.g. $4 \mathrm{~Hz})$. HRV data are typically calculated in time windows of 5 minutes and the FFT calculation can be applied once to this window or to a number of smaller sections (e.g. around 1 minute), for which the spectra afterwards are averaged (Welch's method). Furthermore, it is normal to apply a weighting function (e.g. Hamming window) to the data before the FFT calculation to improve the resolution of the estimated spectrum. Consequently, the frequency analysis using FFT includes several steps with different methodological options. Another method for estimation of the power spectrum is the Lomb-Scargle periodogram (LSP), which unlike the FFT method, can estimate the power spectrum directly from the irregularly sampled RR data thus making the interpolation and resampling step unnecessary [14]. However, the LSP method is like the FFT method sensitive to outliers in the RR data [15]. Recently, a method has been described for robust period detection (RPD) of unevenly sampled data [16]. The method was developed for the analysis of periodicity in data from gene microarrays, but was expected to be useful for other types of uneven sampled biological data. To our knowledge this RPD method has not been used for HRV analysis.

The purpose of this study was to investigate the applicability of the RPD method to HRV analysis with special reference to HR data recorded during everyday life including considerable amount of faulty detected beats.

\section{Methods}

By the RPD method spectral estimates were obtained in 5 minutes periods by the procedure described in ([16], Additional file 1). Briefly, the procedure includes the following steps:

From the actual (unevenly sampled) time of beats $\mathrm{\tau}_{1}, \ldots, \mathrm{T}_{\mathrm{N}}$, a new set of normalized indices $t_{1}, \ldots, t_{N}$ are formed, where

$$
t_{n}=\frac{\left(\tau_{n}-\tau_{1}\right)(\mathrm{N}-1)}{\tau_{N}-\tau_{1}}
$$

Then a set of sine and cosine values are calculated by $\sin \left(2 \pi k t_{n} / N\right), \cos \left(2 \pi k t_{n} / N\right)$ where $\mathrm{k}$ is a frequency index $\mathrm{k}=0,1, \ldots, \mathrm{N} / 2$. These sine and cosine values are used as 
predictor variables for the measured interbeat values $\mathrm{rr}_{1}, \mathrm{rr}_{2}, \ldots, \mathrm{rr}_{\mathrm{n}}$ in a multilinear, robust regression model. From the regression coefficients $A_{s i n, k}$ and $A_{c o s, k}$, the power spectral estimate is calculated by

$$
\frac{N}{4}\left(A_{s i n, k}^{2}+A_{c o s, k}^{2}\right)
$$

for the frequencies

$$
f_{k}=F_{s} \times k / N ; \mathrm{k}=0, \ldots, \mathrm{N} / 2
$$

where $F_{s}$ is the mean sample frequency for the beat time series $\tau_{1}, \ldots, \tau_{N}$.

The robust regression algorithm uses iteratively reweighted least squares with a bisquare weighting function, which assigns less weight to data points causing high residuals and zero weight for outliers (robustfit function called with the parameters Wfun $=$ 'bisquare' and Tune $=4.6851$, [17]). Two cycles of robust regression are executed. First, an initial run is carried out in which the spectral estimates are calculated according to the sequence $\mathrm{k}=0,1, \ldots, \mathrm{N} / 2$. Then the coefficients of the initial spectrum are sorted according to magnitude and a second run of regression is carried out, where the frequencies are processed in the order of descending magnitude. In every step (frequency) of this run the residual from the preceding iteration is used as input i.e. the fitted sinusoidal of the preceding step is subtracted leaving the residual.

Spectral estimates were also obtained by calculation of the FFT and LSP periodograms [18]. The FFT periodogram was obtained by applying a linear interpolation and resampling with a sample frequency of $4 \mathrm{~Hz}$. A Hamming window was applied to $5 \mathrm{mi}$ nutes periods and the FFT algorithm processed for 1024 points. From the spectral estimates calculated by the RPD, FFT and LSP methods, the power was obtained in the low frequency range 0.04-0.15 Hz (LF) and the high frequency range 0.15-0.4 Hz (HF), after which the LF/HF ratio was calculated and logarithmic transformed.

The data used in this study included HR measurements obtained by the Actiheart monitor, which can record and store IBI values for 440.000 beats using two standard ECG electrodes adhered at the V1/V2 and V4/V5 positions, respectively. The beats are detected from signals with a sample frequency of $128 \mathrm{~Hz}$, and IBI values are obtained with a resolution of $1 \mathrm{~ms}$ using an interpolation algorithm [19,20].

IBI data were obtained for 221 subjects (92 females and 129 males in the age of 18-65 years), mainly blue collar workers, for one day of measurements including work hours, leisure time and sleep. The measurements were divided into 5 minutes periods and processed by an algorithm for detection of ectopic beats and artefacts. In the literature abnormal beats are typically defined as beats for which the IBI value deviate more than e.g. $20 \%$ of the previous normal beat [21]. In this study beats were classified as abnormal if IBI values deviated more than 15\% (ectopic strength) from neighboring normal beats.

For error free 5 minutes periods a distortion procedure was carried out that randomly applied errors to beats (ectopic distortion) and groups of beats up to an error rate of $20 \%$. The type of ectopic distortion applied was similar to the real ectopic beats and artifacts generally found in the recordings. A random number/set of single beats or all beats in a random cluster could be displaced with a random value in the interval $\pm 15-40 \%$ or a beat could be excluded. Also, an ectopic distortion scheme was applied 
where all beats, which were selected for distortion, were just deleted from the IBI time series. Finally, a special distortion scheme was applied in which the randomly selected beats (as before) were displaced with exactly $+15 \%$ or $-15 \%$ in order to induce ectopic distortions just at the threshold level for error detection.

From the error free periods power spectra were estimated by RPD, FFT and LSP methods before and after applying the different distortion schemes. Then the logarithmic transformed LF/HF power ratios were calculated and comparisons made by means of linear regression between LF/HF values of the error free and distorted IBI series as a function of the distortion rate.

The very low frequency range (VLF: $0.003-0.04 \mathrm{~Hz}$ ) were not included in the data comparison, so to increase the stationarity of the HR data most of the VLF frequencies were removed before calculation of the spectra. This was achieved by calculating the mean value of a 0.5 minute running window of the IBI values and subtracting the mean from every single IBI value.

By the calculation of spectral estimates it is an assumption that the data are stationary, which means that both the mean and variance should be approximately constant throughout the 5 minutes. To validate these assumptions a test for equal variance was done by splitting the 5 minute periods into 5 sections and performing a Brown-Forsythe test for equal variance on these sections [22]. All calculations were performed using the Matlab programing tool.

\section{Results}

The data set consisted of all 5 minutes periods from 221 subjects monitored for HR during 1 day. Approximately $50 \%$ of the 5 minutes periods were without abnormal beats according to the criterion that normal IBI intervals should not deviate more than $15 \%$ from its neighboring intervals. Thirty percent of the 5 minutes periods were found to include errors but with an amount less than $10 \%$. Figure 1 shows an example of a 5 minutes period without abnormal beats that has been processed by the ectopic distortion algorithm to induce random errors of varying ectopic strength.

Figure 2 shows a plot of the LF/HF ratio of power spectra estimated from IBI series where approximately $1 \%$ of the IBIs have been changed corresponding to a displacement of the beats with a distance of $\pm 15-40 \%$ to its neighbors. The LF/HF ratio for the ectopic distorted IBI series are compared with the undistorted series calculated by the RPD, FFT and LSP methods. It is evident that the small amount of distorted beats (typically $1-3$ beats in an 5 minutes period, $1 \%$ of distorted IBIs corresponds to $0.5 \%$ distorted beats) have a large influence on the FFT and LSP calculated periodogram and very little influence on the RPD results.

Figure 3 shows the results of standard, linear regression between log transformed LF/HF ratios for IBI series with ectopic distorted beats (ectopic strength $\pm 15-40 \%$ ) and removed beats with distortion rates up to $20 \%$ compared to the undistorted series for the three different methods. For a distortion rate of 20\% the RPD method shows a decrease in goodness of fit $\left(R^{2}\right)$ to 0.96 if the distorted beats are removed and to approximately 0.90 if the distorted beats are included in the calculation. Similarly, there is a decrease in the regression coefficient $\mathrm{p} 1$ to 0.93 and 0.87 , respectively; however, the intercepts $\mathrm{p} 0$ are very close to zero. The LSP method demonstrates slightly lower $\mathrm{R}^{2}$ values and considerably lower coefficient values than the RPD methods when the 

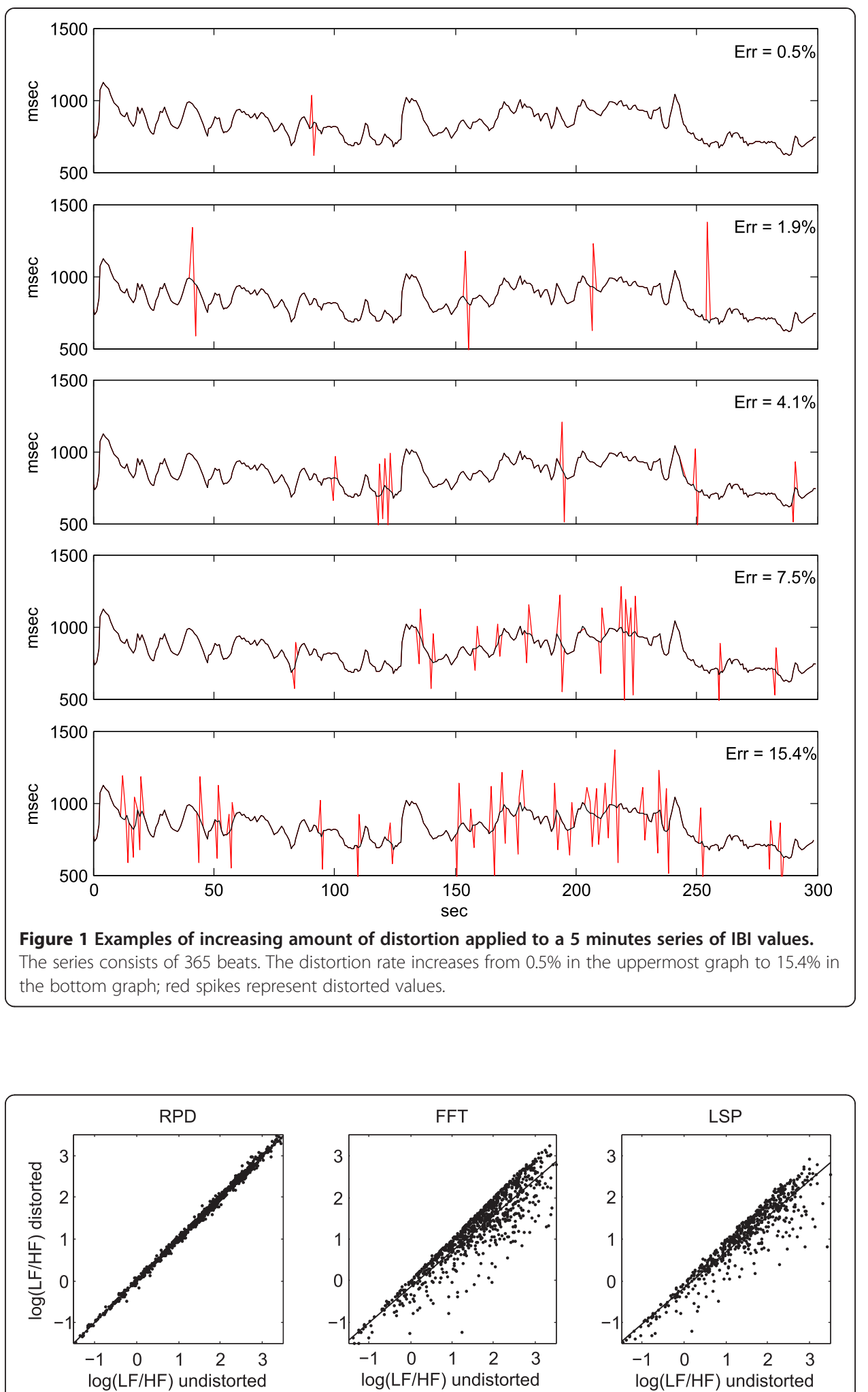

Figure 2 Log transformed LF/HF ratios for distorted IBI series plotted versus undistorted series.

Power spectra were estimated by the RPD, FFT and LSP methods and log transformed LF/HF ratio calculated for 904 five minutes periods with a distortion rate between $0.5 \%$ and $1.5 \%$. Regression for RPD: $\log (Y)=0.996 \cdot \log (X)+0.002, R^{2}=0.998$; regression for $F F T: \log (Y)=0.755 \cdot \log (X)-0.364, R^{2}=0.582$; regression for LSP: $\log (Y)=0.738 \cdot \log (X)-0.520, R^{2}=0.603$ 


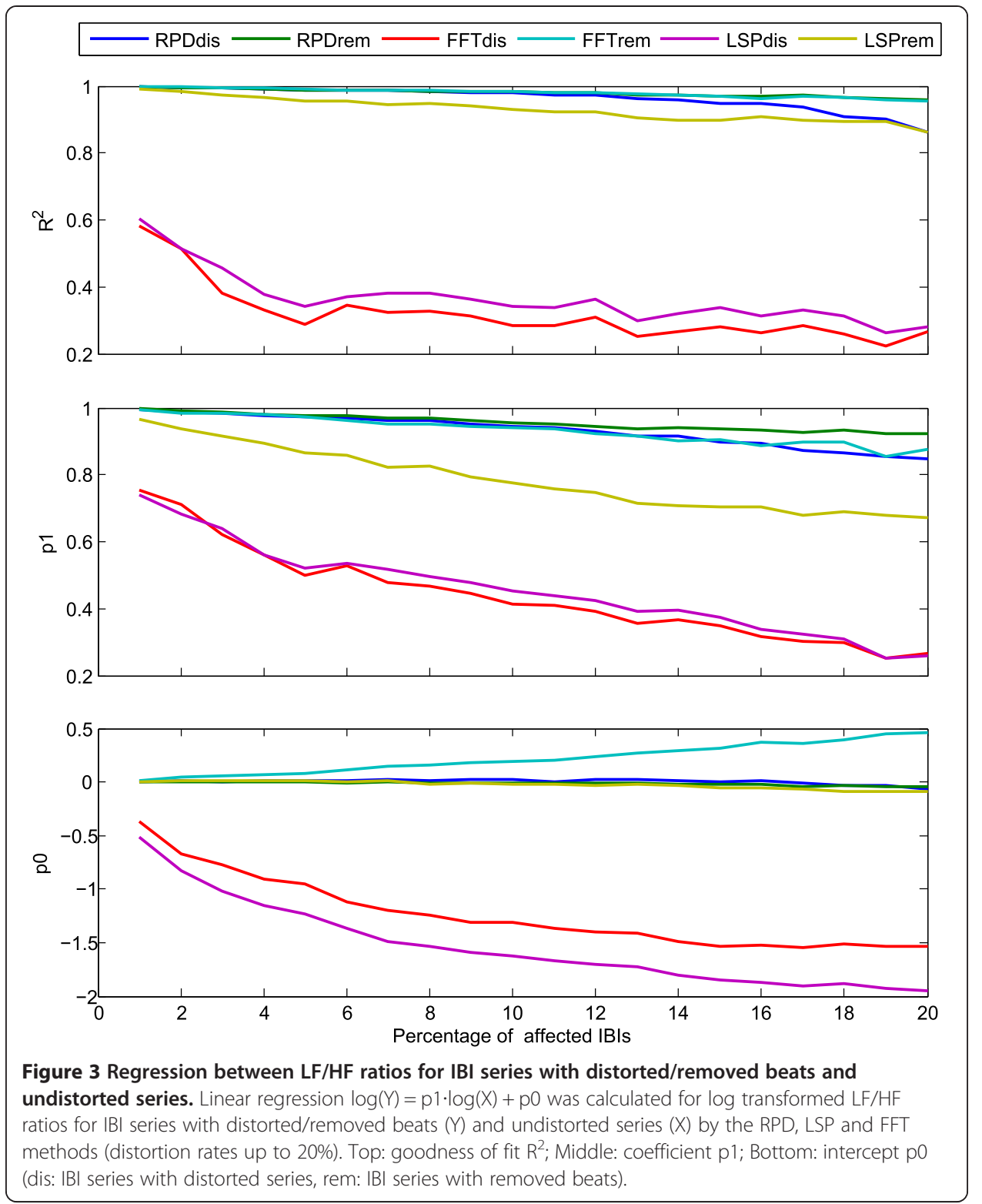

ectopic beats were removed. There are no differences in $R^{2}$ values between the RPD and FFT method if the distorted beats are removed and the spectra are estimated from the remaining IBIs; also the coefficient values for the FFT method approximate those of the RPD methods, however, the intercept values are increasing steadily. For the FFT and LSP method both the goodness of fit and regression coefficients are considerable below 1.0 and the intercept values below -0.5 , when ectopic beats are included in the analysis. Table 1 lists the regression parameters for a distortion rate of $10 \%$ read from Figure 3, and some examples of the deviations between the methods calculated for selected LF/HF values in the range 0.1-10 are shown in Table 2.

The results obtained when the ectopic distortion strength is precisely $\pm 15 \%$, are shown if Figure 4. It appears that the FFT and LSP methods are strongly affected by this level of distortion though there is some improvement compared to the results for 
Table 1 Results of linear regression between LF/HF ratios for IBI series with distorted/ removed beats and undistorted series

\begin{tabular}{ccccccc}
\hline Regression parameter & RPDdis & RPDrem & LSPdis & LSPrem & FFTdis & FFTrem \\
\hline$R^{2}$ & 0.98 & 0.98 & 0.34 & 0.93 & 0.28 & 0.98 \\
p1 & 0.94 & 0.96 & 0.46 & 0.78 & 0.42 & 0.95 \\
p0 & 0.02 & -0.01 & -1.61 & -0.02 & -1.32 & 0.19 \\
\hline
\end{tabular}

Regression parameter values for the linear regression $\log (\mathrm{Y})=\mathrm{p} 1 \cdot \log (\mathrm{X})+\mathrm{p} 0$ between $\log$ transformed $\mathrm{LF} / \mathrm{HF}$ ratios for $\mathrm{IBI}$ series with distorted/removed beats $(\mathrm{Y})$ and undistorted series $(\mathrm{X})$ by the RPD, LSP and FFT methods. The distortion rate was $10 \%$. 'dis' denotes series with distorted beats and 'rem' denotes series with removed beats.

ectopic distortions beyond $\pm 15 \%$. However, the RPD method performs well for distortion strengths of $\pm 15 \%$ for error rates up to approximately $10 \%$.

A total of 29359 five minutes periods were found to be error free. In $81 \%$ of these periods the hypothesis of equal variance throughout the period was rejected $(\mathrm{p}=0.05)$, and in only $0.4 \%$ of the periods equal variance was found $(\mathrm{p}=0.95)$. For the periods with the equal variance the regression between $\log$ transformed LF/HF ratios for FFT and RPD periodograms showed a $R^{2}$ value of 0.96 and the coefficients $(\mathrm{p} 0, \mathrm{p} 1)=$ $(0.28,0.93)$, while for the periods without equal variance $R^{2}=0.79$ and $(\mathrm{p} 0, \mathrm{p} 1)=$ $(0.47,0.83)$. Similar regression calculations for stationary periods of log transformed $\mathrm{LF} / \mathrm{HF}$ ratios for LSP and RPD periodograms showed a $\mathrm{R}^{2}$ value of 0.98 and $(\mathrm{p} 0, \mathrm{p} 1)=$ $(-0.02,0.95)$, while for the periods without equal variance $R^{2}=0.89$ and $(\mathrm{p} 0, \mathrm{p} 1)=$ $(0.26,0.83)$.

\section{Discussion}

This study compared spectral estimates obtained by a new method using a robust period detection to the traditional FFT method and the Lomb-Scargle method. Error free 5 minutes periods of heart rate data were artificially distorted by displacing randomly selected beats by $\pm 15-40 \%$. Periodograms were estimated by all three methods for the basal error free periods, periods with distorted beats and periods with the distorted beats removed. The RPD method demonstrated good performance compared to the FFT and LSP method when calculating the low to high frequency ratio of the periodogram. For example, when using the RPD method the LF/HF estimates are almost identical for error-free periodograms and for periodograms with an error rate of $10 \%$ (of IBI values). RPD with removing abnormal beats resulted in deviations from $+9 \%$

Table 2 Examples of differences (percent) of LF/HF ratios for IBI series with distorted/ removed beats compared to undistorted series

\begin{tabular}{ccccccc}
\hline \multirow{2}{*}{ LF/HF for undistorted series } & \multicolumn{3}{c}{ LF/HF differences for series with distorted/removed beats } \\
\cline { 2 - 7 } & RPDdis & RPDrem & LSPdis & LSPrem & FFTdis & FFTrem \\
\hline 0.1 & $17 \%$ & $9 \%$ & $-31 \%$ & $63 \%$ & $2 \%$ & $36 \%$ \\
0.3 & $10 \%$ & $4 \%$ & $-62 \%$ & $28 \%$ & $-46 \%$ & $28 \%$ \\
3 & $2 \%$ & $-1 \%$ & $-80 \%$ & $-2 \%$ & $-73 \%$ & $21 \%$ \\
10 & $-4 \%$ & $-5 \%$ & $-89 \%$ & $-23 \%$ & $-86 \%$ & $14 \%$ \\
\hline
\end{tabular}

The differences are calculated by the regression parameter values $\mathrm{p} 1$ and $\mathrm{p} 0$ in Table 1 . The distortion rate was $10 \%$. 'dis' denotes series with distorted beats and 'rem' denotes series with removed beats. 


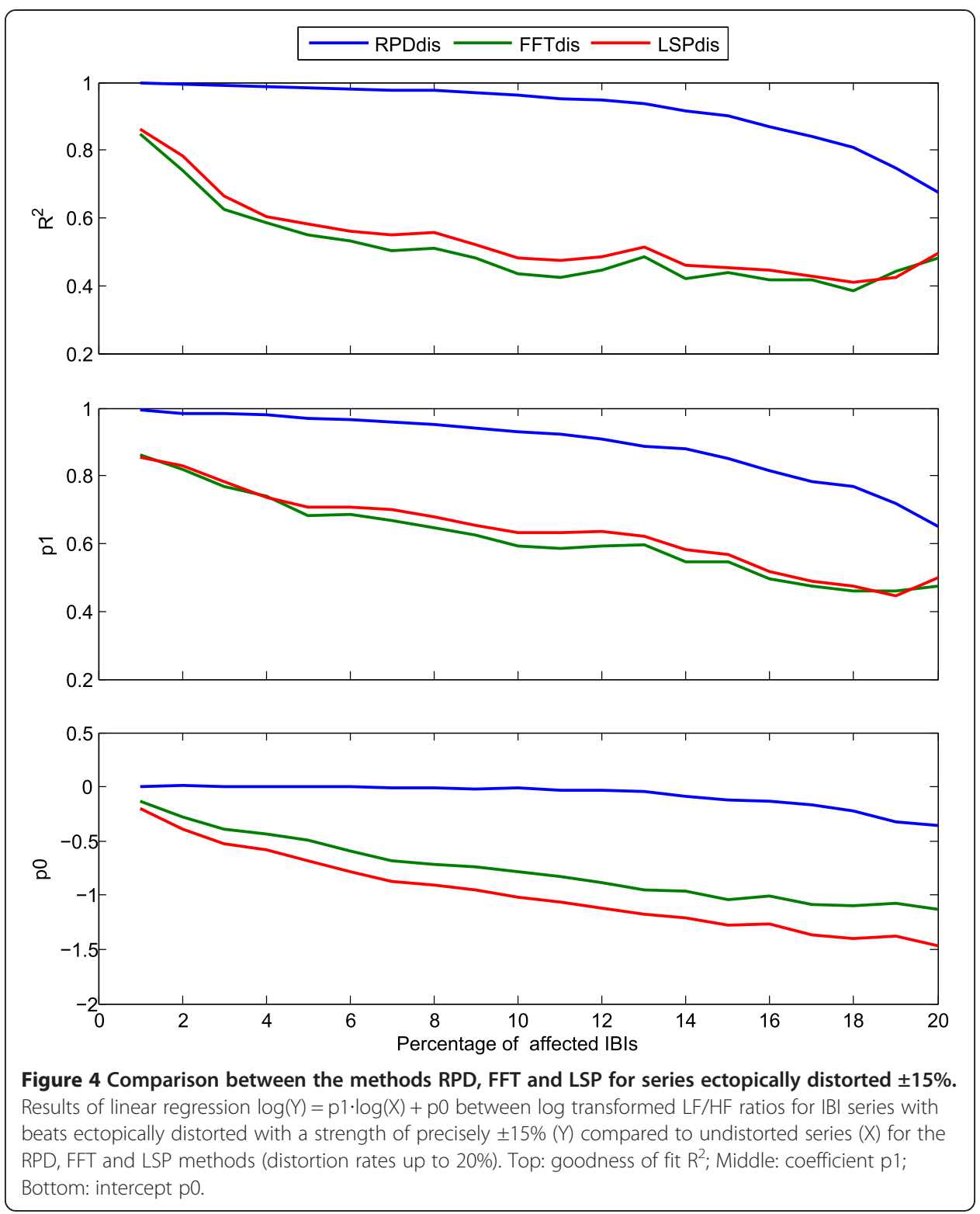

to $-10 \%$ for LF/HF ratios in the range $0.1-10$, and without removing abnormal beats, deviations were from $+17 \%$ to $-11 \%$ (Table 2). Similarly, for the FFT method with removed (and linear interpolated) abnormal beats, the deviations were from $+36 \%$ to $+5 \%$. The occurrence of abnormal beats in heart rate data for periodogram estimation by the FFT and LSP methods result in the well-known underestimation of the LF/HF ratio, because of the high levels of HF power associated with peaks in the tachogram [22]. The deviation for the LSP method with removed abnormal beats (10\% error rate) was in the range $+63 \%$ to $-41 \%$, which is higher than expected as the LSP method is reported to be superior to the FFT method [23]. The reason for the poorer agreement of the LSP method is not known, but it could be speculated that the data of this study include real heart rate recordings, for which the requirement of stationarity very often cannot be fulfilled, while other studies of the LSP method mainly used 
simulated, stationary heart rate data. For higher error rates the RPD method with removing the abnormal beat performs better than the RPD method without removing the abnormal beats. For example for a an error rate of $20 \%$ the RPD method with removing abnormal beats yield deviations of $+13 \%$ to $-18 \%((\mathrm{p} 0, \mathrm{p} 1)=(-0.04,0.93))$, while without removing the abnormal beats, the deviation are $+32 \%$ to $-34 \%((\mathrm{p} 0, \mathrm{p} 1)=(-0.07,0.85))$.

For error rates up to approximately 10\% the RPD method performed well for an ectopic distortion equal to $\pm 15 \%$, while the FFT and LSP were strongly affected. Beats with an ectopic distortion of $\pm 15 \%$, will just remain undetected by the procedure for finding and removing abnormal beats and artifacts, and these faulty beats have a large impact on the FFT and LSP methods. This clearly demonstrates the robustness and benefit of the RPD method compared to the FFT and LSP method, since no procedure for removing faulty beats is perfect; generally, there will be a tradeoff between detection of greatest possible number of abnormal beats and not to eliminate too many normal beats.

In theory the calculation of spectral estimates (and correlation functions) are based on the assumption of stationary data, which means that mean and variance do not vary significantly (weak stationarity). The applied 0.5 minutes mean subtracting procedure will to some degree ensure that the mean do not vary significantly, so the stationarity would mainly depend on approximately equal variance throughout the 5 minutes period. Only very few periods $(0.4 \%)$ were found to meet an equal variance criteria set up in this study and accordingly to show an approximate stationarity. There was a better agreement between the FFT and RPD methods for these periods $\left(R^{2}=0.96\right.$, $(\mathrm{p} 0, \mathrm{p} 1)=(0.28,0.93))$ than between periods without equal variance and accordingly non-stationary $\left(\mathrm{R}^{2}=0.79,(\mathrm{p} 0, \mathrm{p} 1)=(0.47,0.83)\right)$. The regression result for the stationary periods corresponds to an overestimation of the LF/HF ratio by $13 \%$ to $55 \%$ for ratios between 0.1 and 10 for the FFT method compared to the RPD method. This supports the validity of the present RPD method, since it reported that the FFT method with linear resampling can overestimate the LF/HF ratio by $50 \%$ [22]. A similar comparison between the LSP and RPD method for the stationary periods yielded LF/HF ratios for the LSP method to be within $+10 \%$ to $-14 \%$ of the RPD LF/HF ratio in the range 0.1 to 10 $\left(\mathrm{R}^{2}=0.98,(\mathrm{p} 0, \mathrm{p} 1)=(-0.02,0.95)\right)$, so this shows a good agreement supporting the validity of the RPD method. However, for non-stationary recordings the same differences between the methods were from $+92 \%$ to $-12 \%\left(R^{2}=0.89,(p 0, p 1)=(0.26,0.83)\right)$. The poorer agreement between the methods for the non-stationary periods is not surprising because of the violated stationarity assumption. We have included all periods both stationary and non-stationary in the calculations presented in Figures 2 and 3, since practical studies reported in the literature rarely elaborate on this issue.

One drawback of the RPD method is the very time consuming calculations of the periodogram compared to the LSP and FFT methods; the calculation of a periodogram for a 5 minutes period takes around $1 \mathrm{sec}, 40 \mathrm{msec}$ and $1 \mathrm{msec}$ for the RPD, LSP and FFT methods, respectively, executed as a Matlab script by a standard PC. However, for most offline applications this might not be an issue.

\section{Conclusion}

The RPD method demonstrated a superior capacity for estimation of the low to high frequency power ratio of the periodograms for heart rate data with ectopic beats and 
artifacts compared to the FFT and LSP method. Especially, a few marginally ectopic beats typically not recognized by error detecting procedures, have very little influence on the RPD result while seriously affecting the FFT and LSP methods. For error free and stationary heart rate data the RPD showed results similar to the LSP method, and to FFT method, when taking into account the inherent low-pass filtering characteristic of the FFT method.

\section{Additional file}

Additional file 1: Matlab code for robust period detection.

\section{Abbreviations}

RPD: Robust periodic detection; IBI: Interbeat interval; HRV: Heart rate variability; FFT: Fast fourier transform; LSP: Lomb-scargle periodogram; HF: High frequency; LF: Low frequency; VLF: Very low frequency; dis: Distorted; rem: Removed.

\section{Competing interests}

The authors declare that they have no competing interests.

\section{Authors' contributions}

JHS: concept, analysis and drafting of manuscript. JK: drafting of manuscript. Both authors read and approved the final manuscript.

\section{Acknowledgements}

We thank Dr Miika Ahdesmäki for support in finalizing the manuscript.

Received: 16 June 2014 Accepted: 19 September 2014

Published: 23 September 2014

\section{References}

1. Kraus U, Schneider A, Breitner S, Hampel R, Rückerl R, Pitz M, Geruschkat U, Belcredi P, Radon K, Peters A: Individual daytime noise exposure during routine activities and heart rate variability in adults: a repeated measures study. Environ Health Perspect 2013, 121:607-612.

2. Hautala AJ, Karjalainen J, Kiviniemi AM, Kinnunen H, Mäkikallio TH, Huikuri HV, Tulppo MP: Physical activity and heart rate variability measured simultaneously during waking hours. Am J Physiol Heart Circ Physiol 2010, 298:H874-H880.

3. Pumprla J, Howorka K, Groves D, Chester M, Nolan J: Functional assessment of heart rate variability: physiological basis and practical applications. Int J Cardiol 2002, 84:1-14.

4. Pagani M, Lombardi F, Guzzetti S, Rimoldi O, Furlan R, Pizzinelly P, Sandrone G, Malfatto G, Dell'Orto S, Piccaluga E: Power spectral analysis of heart rate and arterial pressure variabilities as a marker of sympatho-vagal interaction in man and conscious dog. Circ Res 1986, 59:178-193.

5. Montano N, Ruscone TG, Porta A, Lombardi F, Pagani M, Malliani A: Power spectrum analysis of heart rate variability to assess the changes in sympathovagal balance during graded orthostatic tilt. Circulation 1994 90:1826-1831.

6. Malliani A, Lombardi F, Pagani M: Power spectrum analysis of heart rate variability: a tool to explore neural regulatory mechanisms. Br Heart J 1994, 71:1-2.

7. Pal GK, Chandrasekaran A, Hariharan AP, Dutta TK, Pal P, Nanda N, Venugopal L: Body mass index contributes to sympathovagal imbalance in prehypertensives. BMC Cardiovasc Disord 2012, 12:54

8. Lucini D, Riva S, Pizzinelli P, Pagani M: Stress management at the worksite. Reversal of symptoms profile and cardiovascular dysregulation. Hypertension 2007, 49:291-297.

9. Vinik Al, Maser RE, Ziegler D: Autonomic imbalance: prophet of doom or scope for hope? Diabet Med 2011 28:643-651.

10. Clifford GD, Azuaje F, McSharry PE: Advanced Methods and Tools for ECG Data Analysis. Boston: Artech House, Inc; 2006.

11. Li H, Kwong S, Yang L, Huang D, Xiao D: Hilbert-Huang Transform for Analysis of Heart Rate Variability in Cardiac Health. IEEE/ACM Trans Comput Biol Bioinform 2011, 8:1557-1567.

12. Storck N, Ericson M, Lindblad LE, Jensen-Urstad M: Automated computerized analysis of heart rate variability with digital filtering of ectopic beats. Clin Physiol 2001, 21:15-24.

13. Clifford GD, Tarassenko L: Quantifying errors in spectral estimates of HRV due to beat replacement and resampling. IEEE Trans Biomed Eng 2005, 52:630-638.

14. Laguna P, Moody GB, Mark RG: Spectral density of unevenly sampled data by least-square analysis: performance and application to heart rate signals. IEEE Trans Biomed Eng 1998, 45:698-715.

15. Schimmel M: Emphasizing difficulties in the detection of rhythms with Lomb-Scargle periodograms. Biol Rhythm Res 2001, 32:341-346.

16. Ahdesmäki M, Lähdesmäki H, Gracey A, Shmulevich I, Yli-Harja O: Robust regression for periodicity detection in non-uniformly sampled time-course gene expression data. BMC Bioinformatics 2007, 8:233.

17. Matlab Statistics Toolbox. 2013 [http://www.mathworks.se/help/stats/robustfit.html]

18. Savransky D: 2008 [http://www.mathworks.com/matlabcentral/fileexchange/20004-lomb-lomb-scargle-periodogram] 
19. Actiheart User Manual. 2013 [http://www.camntech.com/images/products/actiheart/The_Actiheart_User_Manual.pdf]

20. Kristiansen J, Korshøj M, Skotte JH, Jespersen T, Søgaard K, Mortensen OS, Holtermann A: Comparison of two systems for long-term heart rate variability monitoring in free-living conditions - a pilot study. BioMed Eng Online 2011, 10:27.

21. Clifford GD, MCSharry PE, Tarassenko L: Characterizing artefact in the normal human 24-hour RR time series to aid identification and artificial replication of circadian variations in human beat to beat heart rate using a simple threshold. Comput Cardiol 2002, 29:129-132.

22. Brown MB, Forsythe AB: Robust tests for equality of variances. J Am Stat Assoc 1974, 69:364-367.

23. Clifford GD: ECG Statistics, Noise, Artifacts, and Missing Data. In Advanced Methods and Tools for ECG Data Analysis. Edited by Clifford GD, Azuaje F, McSharry PE. Boston: Artech House, Inc; 2006:55-99.

doi:10.1186/1475-925X-13-138

Cite this article as: Skotte and Kristiansen: Heart rate variability analysis using robust period detection. BioMedical Engineering OnLine 2014 13:138.

\section{Submit your next manuscript to BioMed Central and take full advantage of:}

- Convenient online submission

- Thorough peer review

- No space constraints or color figure charges

- Immediate publication on acceptance

- Inclusion in PubMed, CAS, Scopus and Google Scholar

- Research which is freely available for redistribution

Submit your manuscript at www biomedcentral com/submit 